\title{
Tipping in complex systems: theory, methods and applications
}

\author{
G. Ambika ${ }^{1, a}$ and Jürgen Kurths $s^{2,3}$ \\ 1 Indian Institute of Science Education and Research (IISER) Tirupati, Tirupati 517507, India \\ 2 Potsdam Institute for Climate Impact Research, PO Box 6012 03, 14412 Potsdam, Germany \\ 3 Russia World-Class Research Center "Digital bio design and personalized healthcare", Sechenov First Moscow State \\ Medical University, Moscow 119991, Russia
}

Published online 27 September 2021

(C) The Author(s), under exclusive licence to EDP Sciences, Springer-Verlag GmbH Germany, part of Springer Nature 2021

Many real-world complex systems are observed to undergo sudden transitions in their dynamical states or pattern of behavior and then they are said to tip from one emergent state to another. A few such transitions that can affect humanity in many ways are global changes in climate, earthquakes, hurricanes, abrupt shifts in ecosystems, blackouts in power systems, crashing of financial markets, sudden transitions of mind to depression, and surge of epidemics [1-7]. Such tippings have often a huge impact and are mostly irreversible. They happen mostly due to tiny perturbations to the critical values of one or more of the system's parameters or state variables leading to large qualitative changes in the state of the system.

As we know, complex systems are mostly composed of several interacting subsystems and have several degrees of freedom. The complexity in such systems arises from the nonlinearity in the dynamics of the interacting subsystems, heterogeneity in the pattern of interactions among them and presence of multiple time scales in their dynamics. A few typical cases are coupled patches of vegetation and connected lakes, interacting climate elements, multiscale infrastructure systems, hybrid energy systems, etc. Such systems can be modeled using the framework of complex networks of coupled multi-stable subsystems [8]. In such cases, tipping in one subsystem can result in tipping cascades in the whole system [9]. Then, predicting the tipping by identifying early warning signals and developing strategies for harnessing and preventing cascades are of immediate relevance. In addition, larger scale empirical and modeling efforts to understand the potentials of harnessing social tipping dynamics for global benefits, like mitigating climate changes, are equally important needs of highest actuality.

The tipping is induced in complex systems due to bifurcations, fluctuations or noise near critical states,

\footnotetext{
${ }^{a}$ e-mail: g.ambika@iisertirupati.ac.in (corresponding author)
}

and rate-dependent variations or drift in control parameters of the dynamics. In addition, the appearance of riddled-like basins of attraction and fractality-induced tipping, topological complexity of connections or interactions, etc., are identified as factors leading to critical transitions $[10,11]$. In all these mechanisms or processes underlying the onset of tipping, the role of the inducing factors, and the associated scaling phenomena are highly challenging topics of current research. In this context, several case studies of tipping occurring in diverse fields such as climate and environmental sciences, pandemics, clinical studies, and socio-economy are very illustrative and informative to pursue.

In this special issue, 21 articles are presented that reveal recent trends and directions of research related to tipping in complex systems and the pressing challenges and future perspectives in this area. They are grouped such that we begin with mechanisms or processes and measures of tipping, then move on to control of tipping to end with case studies in diverse areas and applications.

The first six articles focus on mechanisms that induce tipping. Gowthaman et al. [12] report how the competing interaction between the symmetry breaking coupling and the degree of heterogeneity decides the emergent dynamics in a system of coupled Stuart-Landau oscillators and results in a bifurcation-induced tipping to an aging state as heterogeneity is increased. The important question of how anticipation can act as a mediator between the social network of individuals and natural dynamic environment system like pollution, is studied by Mueller et al. [13]. They find that a social tipping can stabilize the environment and prevents a potential socio-ecological collapse. As shown by Verma and Ambika [14], sudden transitions or tipping in a collection of systems can be induced due to multiplexing with another network of systems and in this case the coupling topology influences the nature of transitions on both layers, that are multiplexed. Asir et al. [15] propose how dynamic quorum sensing can lead to discon- 
tinuous transitions to steady states, in various biological and chemical systems. In the article by Arumugam et al. [16], we see rate-induced tipping in a spatially coupled ecological system, leading to the extinction of a metacommunity. In the context of noise-mediated transition, Cabrera et al. [17] report a study on a noisemediated transition, which shows how to avoid an extinguishing transition by a process that is mediated by a coupling between the deterministic and stochastic time scales close to the stability boundary.

Next, a couple of articles on measures that characterize tipping phenomena is presented. The study by Ashwin and Newman [18] is on quantifying rate-dependent tipping between chaotic attractors in terms of tipping probabilities. Helfmann et al. [19] discuss noiseinduced tipping between relevant subsets and pathways in agent-based models. Using two models, they illustrate tipping behavior using Transition Path Theory, that gives a statistical understanding of the tipping paths. The next articles discuss strategies to control tipping which is relevant to avoid transitions with undesirable or harmful consequences. Ma et al. [20] present a new perspective to suppress noise-induced critical transitions away from a desirable state to another contrasting one, by an external linear augmentation method and demonstrate the method for bistable systems and tristable systems. In the article by Ansari et al. [21], the Markov chain Monte Carlo method is used to move an epidemic tipping point by reducing the largest eigenvalues of the adjacency matrix of social interactions. This is illustrated for a SIS/SIR model to show that tipping from an epidemic to a non-epidemic state happens in such degree-homogenized networks.

Next, specific case studies are presented on tipping and related phenomena in a variety of diverse areas: compost bomb instability in the continuum limit, Clarke et al. [22], extreme events in models of traffic flow systems, Gupta and Santhanam [23], spreading processes on social contagion in the Copenhagen network, Donges et al. [24], critical slowing down in Salamander flicker vision, Mehrabbeik et al. [25], basin precipitation from satellite data, Gadhawe et al. [26], instability of dust acoustic waves, Gao and $\mathrm{Wu}$ [27], wave propagation in excitable media, Ramakrishnan et al. [28], mimic-species in community dynamics, Garain [29], and finite-size effects and demographic noise ecosystem dynamics, Majumder et al. [30].

We end with two brief reviews on applications of tipping in two different contexts. An interesting application is to harness the tipping points for logic operations [31], such that the tipping mechanism can serve to construct general purpose computing devices. The tipping points in complex systems have the capability to switch between very different attractors under small changes in a parameter and this is exploited to efficiently and flexibly implement the fundamental logic operations, its reliability being enhanced by moderate noise. The challenges faced in detecting oscillatory instabilities in thermoacoustic systems, in particular for the transition from a chaotic state to limit cycle via a state of intermittency, is reviewed by Pavithran et al. [32]. This review also highlights how the presence of inherent fluctuations in the system during the stable operation can be used to provide warning in real time so that control actions can be initiated to mitigate the effects of the critical transition.

We note that studies related to tipping in complex systems are becoming increasingly data driven, primarily due to the complexity and incomplete understanding of the dynamical processes involved. Hence, the future trends lie along a unified multifold approach incorporating dynamical systems theory, adaptive and multilayer networks and machine learning to encompass comprehensive and effective outcomes.

We would like to thank all the authors whose contributions enriched this special issue. Special thanks to Sandrine Karpe and Sabine Lehr for guidance in editorial procedures.

\section{References}

1. M. Scheffer, Critical Transitions in Nature and Society, vol. 16 (Princeton University Press, 2009)

2. F. Yang, Y. Zheng, F. Jinqiao Duan, S.W. Ling, The tipping times in an Arctic sea ice system under influence of extreme events. Chaos 30, 063125 (2020). https://doi. org $/ 10.1063 / 5.0006626$

3. I.M. Ottoa, J.F. Donges, R. Cremadesc, A. Bhowmik, R.J. Hewitte, W. Lucht, J. Rockström, F. Allerberger, M. McCaffrey, S.S.P. Doe, A. Lenferna, N. Moránm, D.P. van Vuuren, H.J. Schellnhubera, Social tipping dynamics for stabilizing Earth's climate by 2050. Proc. Natl. Acad. Sci. USA 117, 2354-2365 (2020). https:// doi.org/10.1073/pnas.1900577117

4. I. Dobson, B.A. Carreras, V.E. Lynch, D.E. Newman, Complex systems analysis of series of blackouts: cascading failure, critical points, and self-organization. Chaos 17, 026103 (2007). https://doi.org/10.1063/1.2737822

5. A.K. Klose, V. Karle, R. Winkelmann, J.F. Donges, Emergence of cascading dynamics in interacting tipping elements of ecology and climate. R. Soc. Open Sci. 7, 200599 (2020). https://doi.org/10.1098/rsos.200599

6. J.J. Kossakowski, M.C.M. Gordijn, H. Riese, L.J. Waldorp, Applying a dynamical systems model and network theory to major depressive disorder. Front. Psychol. 10, 1762 (2019). https://doi.org/10.3389/fpsyg.2019.01762

7. J.M. Drake, T.S. Brett, S. Chen, B.I. Epureanu, M.J. Ferrari et al., The statistics of epidemic transitions. PLoS Comput. Biol. 15, e1006917 (2019)

8. N. Wunderling, J.F. Donges, J. Kurths, R. Winkelmann, Interacting tipping elements increase risk of climate domino effects under global warming. Earth Syst. Dyn. 12, 601-619 (2021). https://doi.org/10. 5194/esd-12-601-2021

9. B.A. Carreras, V.E. Lynch, I. Dobson, D.E. Newman, Critical points and transitions in an electric power transmission model for cascading failure blackouts. Chaos 12, 985 (2002). https://doi.org/10.1063/1.1505810

10. K.S. Suchithra, E.A. Gopalakrishnan, E. Surovyatkina, J. Kurths, Rate-induced transitions and advanced take off in power systems. Chaos 30, 061103 (2020). https:// doi.org/10.1063/5.0002456 
11. B. Kaszás, U. Feudel, T. Tél, Tipping phenomena in typical dynamical systems subjected to parameter drift. Sci. Rep. 9, 8654 (2019). https://doi.org/10.1038/ s41598-019-44863-3

12. I. Gowthaman, V.K. Chandrasekar, D.V. Senthilkumar, M. Lakshmanan, Symmetry breaking induced tipping to aging. Eur. Phys. J. Spec. Top. (2021). https://doi.org/ 10.1140/epjs/s11734-021-00010-6

13. P.M. Mueller, J. Heitzig, J. Kurths, K. Luedge, M. Wiedermann, Anticipation-induced social tipping: can the environment be stabilised by social dynamics? Eur. Phys. J. Spec. Top. (2021). https://doi.org/10.1140/ epjs/s11734-021-00011-5

14. U.K. Verma, G. Ambika, Tipping induced by multiplexing on two layer networks. Eur. Phys. J. Spec. Top. (2021). https://doi.org/10.1140/epjs/ s11734-021-00116-x

15. M.P. Asir, S. Dixit, M.D. Shrimali, Critical transition influenced by dynamic quorum sensing in nonlinear oscillators. Eur. Phys. J. Spec. Top. (2021). https://doi. org/10.1140/epjs/s11734-021-00012-4

16. R. Arumugam, V.K. Chandrasekar, D.V. Senthilkumar, Rate-induced tipping and regime shifts in a spatial ecological system. Eur. Phys. J. Spec. Top. (2021). https:// doi.org/10.1140/epjs/s11734-021-00139-4

17. J.L. Cabrera, E.D. Gutiérrez, Extinctions in time delayed population maps, fallings and extreme forcing. Eur. Phys. J. Spec. Top. (2021). https://doi.org/10. 1140/epjs/s11734-021-00015-1

18. P. Ashwin, J. Newman, Physical invariant measures and tipping probabilities for chaotic attractors of asymptotically autonomous systems. Eur. Phys. J. Spec. Top. (2021). https://doi.org/10.1140/epjs/ s11734-021-00114-z

19. L. Helfmann, J.H.P. Koltai, J. Kurths, C. Schuette, Statistical analysis of tipping pathways in agent-based models. Eur. Phys. J. Spec. Top. (2021). https://doi. org/10.1140/epjs/s11734-021-00191-0

20. S. Ansari, M. Anvari, O. Pfeer, N. Molkenthin, F. Hellmann, J. Heitzig, J. Kurths, Moving the epidemic tipping point through topologically targeted social distancing. Eur. Phys. J. Spec. Top. (2021). https://doi.org/10. 1140/epjs/s11734-021-00138-5

21. J. Ma, X. Yong, D. Liu, R. Tian, S. Ma, U. Feudel, J. Kurths, Suppression of noise-induced critical transitions, a linear augmentation method. Eur. Phys. J. Spec. Top. (2021). https://doi.org/10.1140/epjs/ s11734-021-00112-1

22. J. Clarke, C. Huntingford, P. Ritchie, P. Cox, The compost bomb instability in the continuum limit. Eur. Phys. J. Spec. Top. (2021). https://doi.org/10.1140/ epjs/s11734-021-00013-3
23. K. Gupta, M.S. Santhanam, Extreme events in NagelSchreckenberg model of traffic flow on complex networks. Eur. Phys. J. Spec. Top. (2021). https://doi.org/ 10.1140/epjs/s11734-021-00016-0

24. J.F. Donges, J. Lochner, J. Heitzig, N. Kitzmann, S. Lehmann, M. Wiedermann, J. Vollmer, Dose-response function approach for detecting spreading processes in temporal network data; exploring social contagion in the Copenhagen networks study. Eur. Phys. J. Spec. Top. (2021). https://doi.org/10.1140/epjs/ s11734-021-00279-7

25. M. Mehrabbeik, R. Ramamoorthy, K. Rajagopal, F. Nazarimehr, S. Jafari, I. Hussaine, Critical slowing down indicators in synchronous period-doubling for salamander flicker vision. Eur. Phys. J. Spec. Top. (2021). https://doi.org/10.1140/epjs/s11734-021-00113-0

26. M.A. Gadhawe, R.K. Guntu, A. Agarwal, Networkbased exploration of basin precipitation based on satellite and observed data. Eur. Phys. J. Spec. Top. (2021). https://doi.org/10.1140/epjs/s11734-021-00017-z

27. D.-N. Gao, W. Jian-Peng, Multi-dimensional instability of dust acoustic waves in magnetized quantum plasmas by two-fluid quantum hydrodynamics model. Eur. Phys. J. Spec. Top. (2021). https://doi.org/10.1140/ epjs/s11734-021-00110-3

28. A. Ramakrishnan, I. Moroz, C. Lic, A. Karthikeyane, K. Rajagopal, Effects of noise on the wave propagation in an excitable media with magnetic induction. Eur. Phys. J. Spec. Top. (2021). https://doi.org/10.1140/ epjs/s11734-021-00115-y

29. K. Garain, Only mimic-species can survive when external noises are added in a ratio-dependent community dynamics model. Eur. Phys. J. Spec. Top. (2021). https://doi.org/10.1140/epjs/s11734-021-00111-2

30. S. Majumder, A. Das, A. Kushal, S. Sankaran, V. Guttal, Finite-size effects, demographic noise, and ecosystem dynamics. Eur. Phys. J. Spec. Top. (2021). https:// doi.org/10.1140/epjs/s11734-021-00184-z

31. K. Murali, S. Sinha, V. Kohar, W.L. Ditto, Harnessing tipping points for logic operations. Eur. Phys. J. Spec. Top. (2021). https://doi.org/10.1140/epjs/ s11734-021-00014-2

32. I. Pavithran, V.R. Unni, R.I. Sujith, Critical transitions and their early warning signals in thermoacoustic systems. Eur. Phys. J. Spec. Top. (2021). https://doi.org/ 10.1140/epjs/s11734-021-00214-w 